\title{
Distance Measurement Systems Using Lasers and Their Applications
}

\author{
João António Santos Dias Fonseca ${ }^{1}$, António Baptista ${ }^{3}, \mathrm{M}^{\mathrm{a}}$ João Martins ${ }^{1} \&$ João Paulo N. Torres ${ }^{2}$ \\ 1 Academia Militar, Lisboa, Portugal \\ 2 Instituto de Telecomunicações, DEEC/ Instituto Superior Técnico, Universidade de Lisboa, Lisboa, Portugal \\ 3 DEEC/ Instituto Superior Técnico, Universidade de Lisboa, Lisboa, Portugal \\ Correspondence: João Paulo N. Torres, Instituto de Telecomunicações, DEEC/ Instituto Superior Técnico, \\ Universidade de Lisboa, Lisboa, Portugal. E-mail: joaotorres@tecnico.ulisboa.pt
}

Received: June 22, 2017

doi:10.5539/apr.v9n4p33
Accepted: July 15, 2017

Online Published: July 24, 2017

URL: https://doi.org/10.5539/apr.v9n4p33

\begin{abstract}
Optical measuring systems came to offer new ways to determine distances, deformations or vibrations through more accurate and greater range methods. Technological progress has allowed a significant improvement of several components, including the optical ones. Thus, the development of essential measurement methods is crucial to keep up with technological advances.

In this paper, the three basic methods of measurement are studied - triangulation, telemetry and interferometry, covering their main applications, advantages and disadvantages, and theoretically substantiating each of the methods. The results of simulation routines for each method are shown along this work. For the triangulation method we made two experiments which demonstrate the functioning of the method in measuring distances and surfaces.

For the telemetry method, experimental setups were studied to apply the method of pulse telemetry and phase comparison telemetry to measure distances,
\end{abstract}

Thus, it is intended to set up the basis for the future development of more complex methods.

Keywords: Interferometry, Optical Measures, Telemetry, Triangulation

\section{Introduction}

\subsection{Introduce the Problem}

In 1960 physicist Theodore Maiman (Martin, 2007) built the first laser, and, since then, applications started being developed to use the new discovery. One of the most important applications, and of greater importance for this work, was the use of lasers in measurement methods.

In the military field, there are several systems that may take advantage of more complex systems of measurement methods. With technology development, the conventional human soldier is being replaced by remotely controlled systems, such as robots, UAVs (Unmanned Aerial Vehicles) and guided missiles. All these systems would improve their performance and robustness with the incorporation of a laser measurement system that would allow them to check how far they are from a particular object or target. Also in the manufacture of military equipment such as tanks, ammunition, weapons, among others, laser deformation measurement systems can be used to verify if the material is able to be used.

\subsection{Evolution of Optical Measurement Systems}

Optical measurement systems are used in various situations, such as deformation measurement of objects, vibration measurement or distance measurement, the latter being the most focused aspect of this paper.

The distance measurement optical methods can be grouped into three categories: triangulation, telemetry, which is based on determining the so-called time-of-flight, and interferometry. In recent years, there has been a considerable progress of optical measurement systems through the understanding of basic physical principles and theoretical information about the detection range (Amann et al., 2001). The development in lasers creation, integrated optical devices, electronic transmitters and receivers has led to new developments in measurement methods. 


\section{Triangulation}

Triangulation is a process that allows to determine distances or positions of objects from considerations based on the geometry of similar triangles. This method has been used around the year $600 \mathrm{BC}$ by the Greek mathematician Thales of Miletus in measuring the height of the Giza pyramids and determining the distance to a ship that was in the sea (Berkovic \& Shafir, 2012).

This method is often used when measuring distances. A triangulation-based optical sensor is a simple example of the application of this method. A collimated laser source (CLS) is used to illuminate the target (it can be an object, a person, etc.) to be measured. The light reflected by the target is detected by a receiving system placed laterally relative to the laser source, constituted for example by a lens and the position sensitive detector (PSD). As it can be seen in Fig. 1, the distance to the target can be determined from the similarity of the formed triangles. Note that these optical sensors, where the laser source and the detector are on the same device, are typically applied for measuring distances between $10 \mathrm{~mm}$ and $1 \mathrm{~m}$ (Berkovic \& Shafir, 2012).

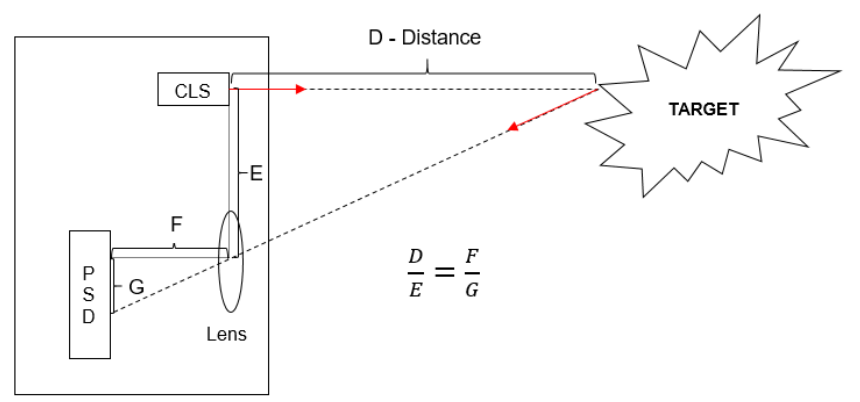

Figure 1. Triangulation Method

In this example, distance D is calculated by (1).

$$
D=\frac{F \times E}{G}
$$

As the distances $\mathrm{E}$ and $\mathrm{F}$ are known, by measuring the $\mathrm{G}$ value (in this case, by the PSD), one calculates the D required distance.

The physical simulation and experience are based on the same scheme shown in Fig. 2, which uses the 5 dotes face of a dice as a target.

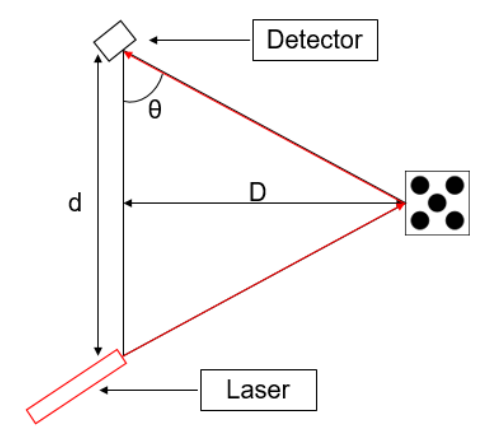

Figure 2. Simulation and experiment scheme

Observing Fig. 2, there is the trigonometric relation of the (2):

$$
\tan \theta=\frac{D}{d / 2}=\frac{2 D}{d}
$$

As distance $d$ is constant, for each measured $\theta$ angle, the wanted $\mathrm{D}$ distance can be determined. The calculation of this distance is shown in (3). 


$$
D=\frac{d \cdot \tan \theta}{2}
$$

For the simulation of the experience based on the triangulation method, a program was created in the software Wolfram Mathematica 10.3 Student Edition.

The simulation shows the conducted experiment with a difference: in the simulation the laser / detector unit is the one that moves in relation to the dice, while in the experiment it is the dice that moves in relation to the laser / detector unit. In theory this difference does not have any interference in the results, since there are no errors associated with the movement of the various elements. However, in the experiment, moving only the dice gives way to fewer errors than moving the laser / detector unit, as it must be ensured that the distance between them is constant.

Since, in theoretical terms, these models are equal and the dice surface will be drawn in a grid, it was decided to vary the position of the detector and laser in the simulation, rather than moving the entire surface of the dice, in order to simplify the representation in the grid.

Figure 3 shows the results of this simulation.

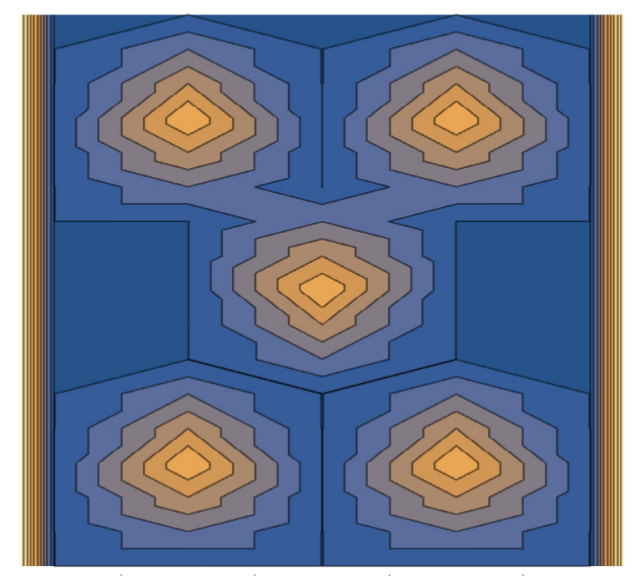

Figure 3. Results of simulation

As it can be observed, the 5 dots of the simulated face were detected.

To prove the theoretical and simulation results, two experiments were made with the triangulation method. The first was a test to check whether the method application worked at a distance of $50 \mathrm{~cm}$ and $70 \mathrm{~cm}$, having a mirror as a target. It has been found that the method resulted in full in the measurement of the mentioned distances, i.e., it was reached almost the maximum laser power focusing the detector, with the angles adapted to the referred distances. Thus, a second experiment was made with a dice at about $70 \mathrm{~cm}$ from the detector. This time, the goal was to scan across the whole 5 dots face of the dice to observe its surface, inserting all distances in a program developed with software Wolfram Mathematica 10.3 Student Edition.

Describing now the physical experiment made, Fig. 4 shows the experiment setup performed.

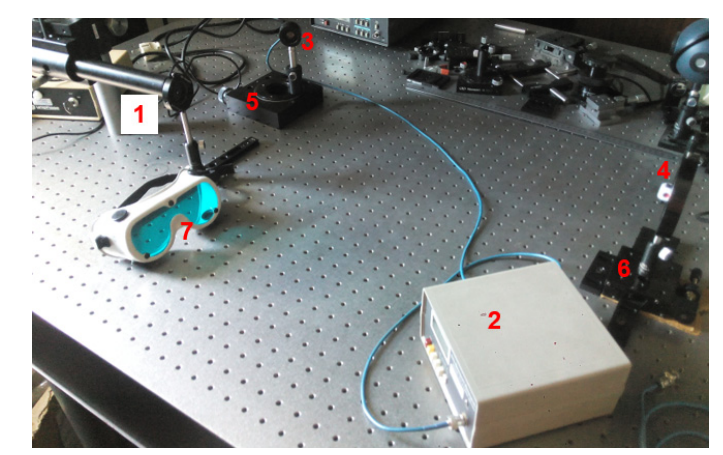

Figure 4. Experiment setup 
In the experimental setup the following materials were used: JDS Uniphase 1100 Series red helium-neon laser model 1101 / P (1), Newport Model 815 optical power meter (2) with detector Model s1818 (3), rotary holder to attach the detector to the Newport optical table (5), movable holder to attach the dice to the Newport optical table (6), dice (4) and goggles (7).

The dice was placed on a movable holder to allow its movement and the dice face corresponding to number 5 was used as a surface to be detected. The experiment consisted of scanning the dice by moving the holder with a micrometric screw. As the experiment requires a constant distance between the detector and the laser (distance $d$ in Fig. 2), it was decided to vary the position of the dice and to keep the position of the detector and the laser constant. In order to measure the angle between the level detector / laser and the level detector / dice (angle $\theta$ in Fig. 2) in any given position of the dice, the laser and the detector were moved in a movable rotary holder with an analog angular scale (useful for measuring angles) until the maximum power is achieved. Note that the laser and the detector move in equal proportion, so that the angle $\theta$ is equal in both. The angles $\theta$ were registered in order to make the distance calculations by (3), using the triangular geometry of the experiment.

All the measured angles were saved in an Excel file, where the calculations of the distance were made, using (3). Fig. 5 shows the result obtained with the triangulation method experiment.

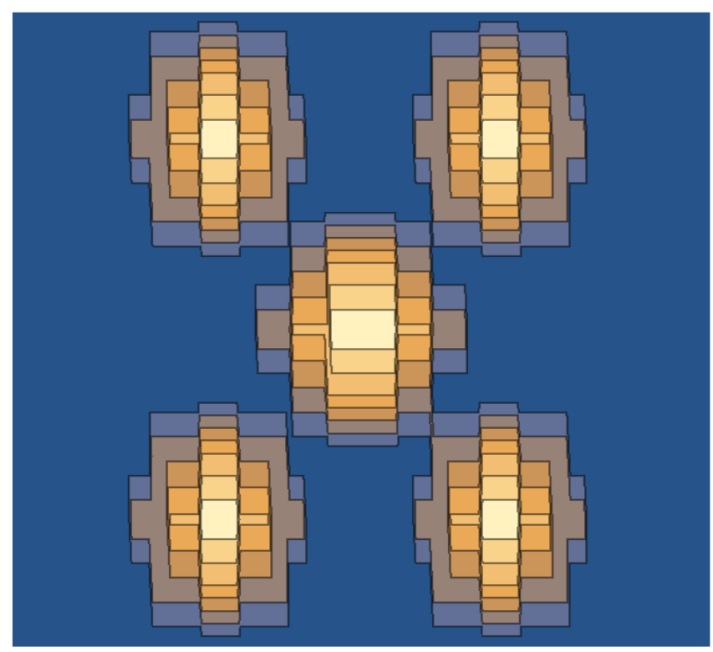

Figure 5. Results obtained with the triangulation method experiment

As it can be seen in Fig. 3 and Fig. 5, simulation and experiment have similar results, proving the success of the experiment performed.

\section{Telemetry}

The distance measurement techniques using the telemetry method were originally used in military applications and topographic surveying applications. There are two basic telemetry methods: the pulsed telemetry method and telemetry method by phase comparison.

\subsection{Pulse Telemetry}

The method pulse telemetry is based on determining the time it takes a power pulse to travel from the transmitter to the target and then back to the detector.

Figure 6 shows a representative scheme of a system that measures distance by the method of pulse telemetry.

As it can be seen in Fig. 6, when the starting signal is received (Start), a counter is activated until the final signal (Stop). After traveling a distance of 2D, the laser beam enters an avalanche photodiode (APD), generating an electrical signal whose voltage is amplified, then acting in the microcontroller, stopping the count. The distance between the object and the transmitter is given by (4), where $\mathrm{f}_{\mathrm{clk}}$ is the clock frequency and $\mathrm{N}$ is the number of digits counted between the initial and the final signal (Nejad \& Olyaee, 2006). 


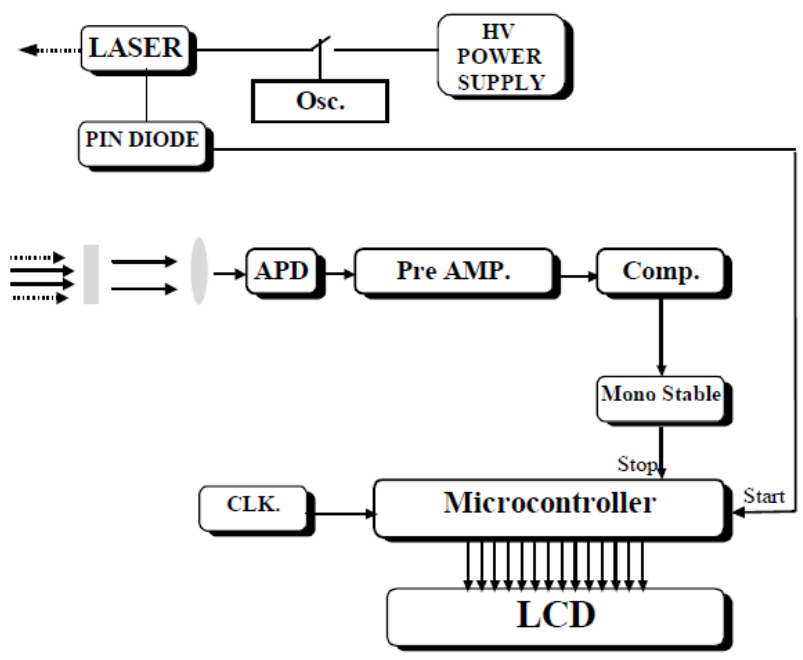

Figure 6 . Scheme using the method of pulse telemetry

$$
D=\frac{N \cdot c}{2 f_{c l k}}
$$

Through clock frequency, it is possible to calculate the time required for the counter to make a count. In the system shown in Fig. 6, the clock frequency is $30 \mathrm{MHz}$. The time (T) spent between each increment in the count is shown in (5).

$$
T=\frac{1}{f_{c l k}}=\frac{1}{30 \times 10^{6}}=33 \text {. (3) } n s
$$

Since $\mathrm{T}$ is calculated, one can determine the measure uncertainty of this system. At the speed of light (c) during this period of time, the distance $\left(D_{\text {inc }}\right)$ traveled by the laser pulse can be calculated using (6).

$$
D_{\text {inc }}=c \times T=10 \mathrm{~m}
$$

Thus, it is concluded that during the path of going and returning, the counting system can make a mistake up to 10 $\mathrm{m}$, so the uncertainty of the maximum measure of distance $D$ is half of this value, i.e., $5 \mathrm{~m}$.

Through (4), the distance to the object is calculated, this being a simple and useful process for long distances, as it has a measurement uncertainty of 5 meters, which for small distances implies a non-negligible relative error.

To simulate the method of pulse telemetry, it was considered that the laser and detector were built in the same place and at the start of the laser pulse emission, the time counting is started at the detector until the receiving of that same impulse. The time count is done considering that there is a clock with $50 \mathrm{MHz}$ frequency to act in a counter that allows this clock frequency.

With a clock frequency of $50 \mathrm{MHz}$, and using (5) one gets a $20 \mathrm{~ns}$ time $T$. Thus, through (6) one gets a $6 \mathrm{~m}\left(D_{\text {inc }}\right)$, i.e., an uncertainty in the maximum extent of $3 \mathrm{~m}$. To develop this program, it was considered that every $6 \mathrm{~m}$ traveled by the laser pulse, i.e., after $20 \mathrm{~ns}$, a counter built in the detector incremented 1 to the count to obtain the value of $N$ and thus calculate the distance by (4).

The scheme principle used in this simulation is similar to that shown in Fig. 6. In the simulation, the scheme is simplified since the start counting, shown in Fig. 6, occurs at the instant the laser pulse starts its journey.

In the simulation, the object was put $50 \mathrm{~m}$ from the laser and detector. In the given results it was found that the object was detected at a distance of $48 \mathrm{~m}$. In this measurement there was an error of $2 \mathrm{~m}$, which is within the $3 \mathrm{~m}$ maximum error mentioned before. This result proves that this method is effective for distances over tens of meters.

Figure 7 shows the scheme used in the experimental assembly. 


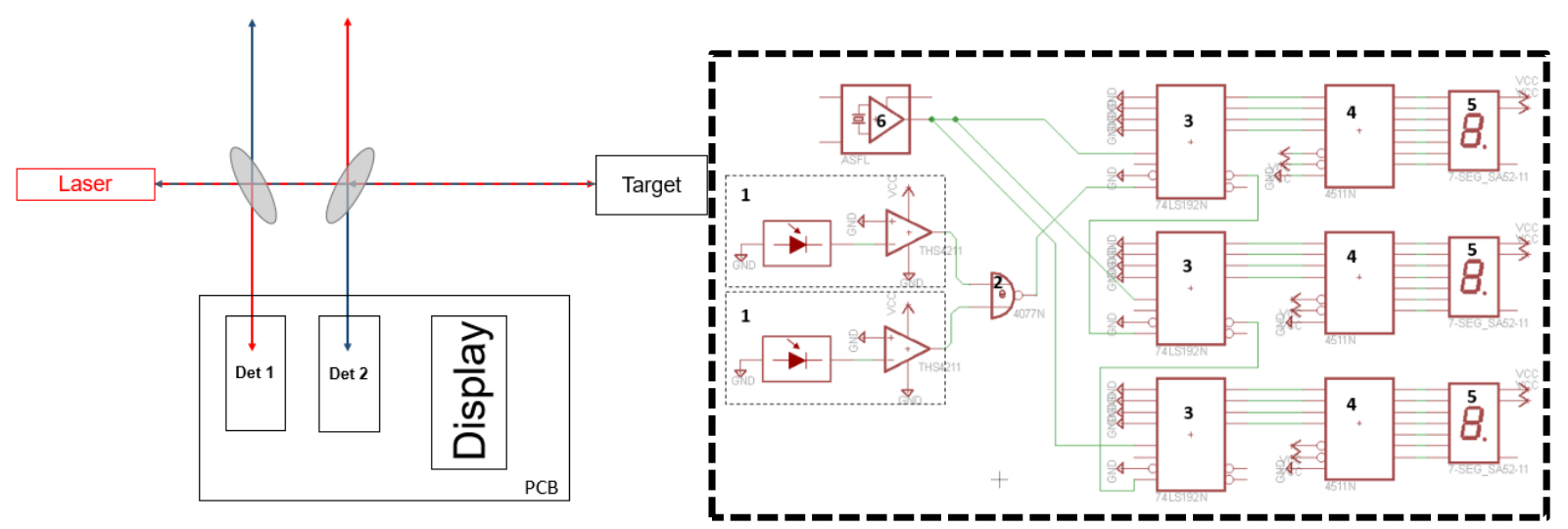

Figure 7. Scheme using the pulse telemetry method

The PCB (Printed Circuit Board) has been designed using the EAGLE 7.2.0 software. This software lets you design the board scheme using integrated circuits that are included in their own libraries or design new integrated circuits if necessary. After the circuit has been designed, the software also allows to pass the elements to a virtual board with real dimensions where you choose the most efficient connections between elements.

The purpose of the PCB is to determine the time-of-flight that the laser pulse takes to travel the distance to the target and back to the board. This time is used, by (4), to determine the distance between the plate and the target, as discussed above. The count starts when the laser pulse focus on the first photodetector and ends when the laser pulse focus on the second photodetector. Observing the count result in displays (value $N$ of (4)), the desired distance was calculated.

\subsection{Phase Comparation Telemetry}

In the method for phase comparison telemetry, a laser beam modulated in intensity or frequency is sent toward the target. In order to perform the phase comparison, a laser reference beam is sent directly to a phase comparison meter. Figure 8 shows a scheme of the method (Hadni, 1993).

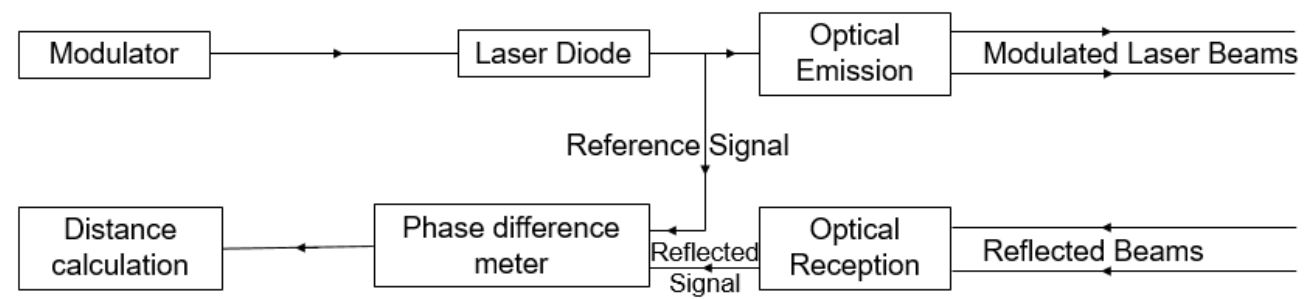

Figure 8. Scheme that uses method for phase comparison telemetry

Being $v_{M}$ the modulation frequency of the beam, the intensity of the emitted beam is shown in (7).

$$
I_{E}=I_{0}\left(1+\cos 2 \pi v_{M} t\right)
$$

Being the signal returning time $2 \mathrm{D} / \mathrm{c}$, where $\mathrm{D}$ is the distance to be determined, the intensity of the reflected beam is shown in (8).

$$
I_{R}=I_{0}\left(1+\cos 2 \pi v_{M}\left(t-\frac{2 D}{c}\right)\right)
$$

Thus, the phase shift of the signal is shown in (9).

$$
\Delta \varphi=4 \pi v_{M} \frac{D}{c}
$$

The distance $D$ is determined by (10). 


$$
D=\frac{c(\Delta \varphi+2 k \pi)}{4 \pi v_{M}}
$$

Being $k$ an integer value, the distance $D$ will always have an uncertainty in this value. To solve the problem, two modulation frequencies were used close enough, so that $k$ does not change. Thus, the distance can be calculated by (11), where $v_{M}$ and $v_{M}$ ' are the used modulation frequencies.

$$
D=\frac{c\left(\Delta \varphi_{0}+2 k \pi\right)}{4 \pi v_{M}}=\frac{c\left(\Delta \varphi_{0}{ }^{\prime}+2 k \pi\right)}{4 \pi v_{M}{ }^{\prime}}
$$

Thus, it is possible to eliminate $\mathrm{k}$ value from the expression. The distance $D$, which is to be determined, is calculated by (12).

$$
D=\frac{c\left(\Delta \varphi_{0}-\Delta \varphi_{0}{ }^{\prime}\right)}{4 \pi\left(v_{M}-v_{M}{ }^{\prime}\right)}
$$

To determine the error associated with this calculation, the author indicates that the $\Delta \varphi$ phase difference has a 0.7 mrad error. Through (10), with a $30 \mathrm{MHz}$ modulation frequency, it was obtained a $0.557 \mathrm{~mm}$ measurement error.

To simulate the method for phase comparison telemetry a program was created, where an object located $50 \mathrm{~cm}$ from the detector was used. In this program, the distance between laser and detector is much lower than the distance between them and the object, simulating a sensor with a built-in laser and detector.

As a result of the simulation, the object was detected at a distance of exactly $50 \mathrm{~cm}$. This method showed a result without any measurement error. As already mentioned, the measurement error associated with this method occurs due to an error when determining the phase difference. In this program this error does not happen, because the phase comparison is calculated using formulas instead of a physical phase comparator.

Figure 9 shows the scheme used in the experimental assembly.
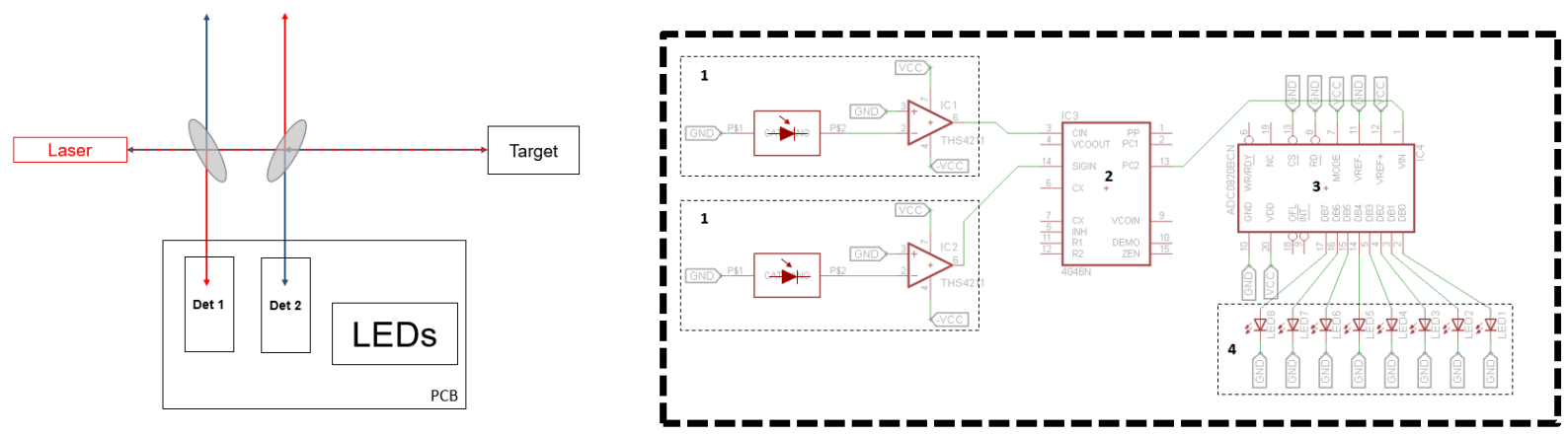

Figure 9. Scheme using the method for phase comparison telemetry

The PCB has been designed using the EAGLE 7.2.0 software.

The purpose of the PCB is to determine the phase difference between a laser impulse that travels a known distance and a laser impulse that is reflected at a target. This phase difference is used, by (10), to determine the distance between the plate and the target, as discussed above.

\section{Interferometry}

With the appearance of several space missions based on instruments that consisted of independent satellites working cooperatively, the importance of optical metrology increased significantly, as it involves measurement uncertainties between $10 \mu \mathrm{m}$ and $10 \mathrm{~nm}$ (A Cabral \& Rebordão, 2015).

To achieve this resolution, the appropriate method is interferometry. In this method, the distance is measured by phase difference between two waves, where one of the waves is known. For the application of this method several interferometers were invented, among which there are the Michelson interferometer, the Fabry-Perot interferometer and Mach-Zehnder interferometer. This paper main focus was given to Michelson interferometer.

Michelson interferometer was the first to be invented (resulting from the Michelson-Morley experiment in 1887). It consists in dividing the light beam that travels two paths. After reflection on the target, the reflected signals are 
recombined in a detector, producing an interference pattern. Fig. 10 shows the operation of this interferometer, where the emitting source is a laser diode (Kikuta, Iwata, \& Nagata, 1986).

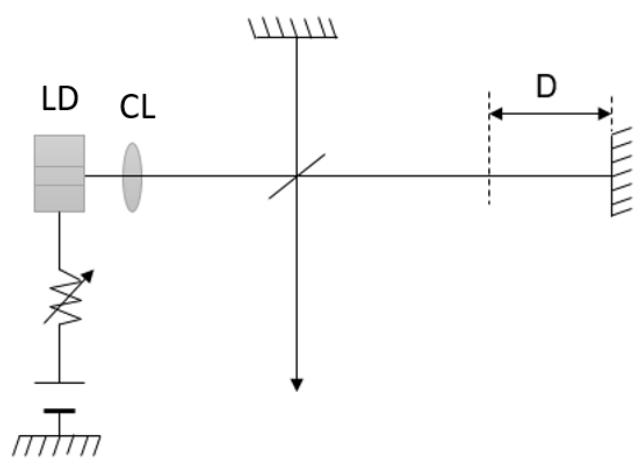

Picture 10. Michelson interferometer

In this example, the light emitted by the laser diode (LD) is collimated by a lens and directed to a Michelson interferometer. The phase difference between the two beams is determined at the detector, and from this difference, the size difference of the two arms of the interferometer was calculated. This method is mainly used to measure distances, so it is important for this work.

The difference $L$ between the paths of the two interferometer arms is twice the distance $D$, that is to be measured. The corresponding phase difference is calculated by (13.1), where $\lambda$ is the wavelength of the laser light (Kikuta, Iwata, \& Nagata, 1986).

$$
\Delta \phi=\frac{2 \pi L}{\lambda}
$$

When the wavelength is changed from $\lambda$ to $\lambda+\Delta \lambda$ by means of a change in the laser injection current, the phase difference also undergoes a change as it is shown in (13.2).

$$
\Delta \phi=2 \pi L\left(\frac{1}{\lambda}-\frac{1}{\lambda+\Delta \lambda}\right)
$$

If the change of wavelength is minimal compared to the wavelength, the phase difference can be approximated by (13.3).

$$
\Delta \phi \cong 2 \pi L \frac{\Delta \lambda}{\lambda^{2}}
$$

As $\lambda$ and $\Delta \lambda$ are known values, the desired distance can be determined by means of the difference between the paths of the two arms of the interferometer by (14).

$$
D=\frac{L}{2} \cong \frac{\Delta \phi \lambda^{2}}{4 \pi \Delta \lambda}
$$

It is necessary to take into account that the wavelength of the laser light varies with the injection current and the temperature. Normally, the light wavelength increases $0.005 \mathrm{~nm}$ per each current $\mathrm{mA}$ at a wavelength of $800 \mathrm{~nm}$ and it increases $0.04 \mathrm{~nm}$ per ${ }^{\circ} \mathrm{C}$.

To simulate the interferometric method a program was created, in which an object located $10005 \mu \mathrm{m}$ from the detector was used. Simulating that we are before a Michelson interferometer, the laser pulse is divided into two pulses, in which one goes toward the target from one of the arms and the other goes out toward a reference from the other arm.

As a result of the simulation, the object was detected at a distance of exactly $10005.0108 \mu \mathrm{m}$ from the detector. The distance that the object was from the detector was $10005 \mu \mathrm{m}$. The $0.0108 \mu \mathrm{m}$ error has nothing to do with the method of interferometry, but with approaches made by the software during the calculation process. Errors occur in this method when there are wavelength changes caused by changes in the laser injection current and / or temperature changes. These factors are not present in the software, so the errors in this program are not expected. 


\section{Comparation of the three Mthods}

Table 1 shows a comparison of the three methods in terms of the distance they can measure accurately

Table 2 shows a comparison of the three methods in the essential formulas.

Table 3 shows a comparison of the three methods related to the measurement uncertainty.

Table 1. Distance comparison

\begin{tabular}{cc}
\hline Methods & Distance \\
\hline Triangulation & $10^{-5}-10^{1}$ \\
Pulse Telemetry & $1-10^{3}$ \\
Phase Comparison Telemetry & $10^{-3}-1.5$ \\
Interferometry & $10^{-9}-10^{-5}$ \\
\hline
\end{tabular}

Table 2. Essential formulas comparison

\begin{tabular}{cl}
\hline Methods & Distance \\
\hline Triangulation & $A C=\frac{\mathrm{AB} \cdot \sin \beta}{\sin (\alpha+\beta)}$ \\
Pulse Telemetry & $D=\frac{N \cdot c}{2 f_{c l k}}$ \\
Phase Comparison Telemetry & $\mathrm{D}=\frac{c\left(\Delta \varphi_{0}-\Delta \varphi_{0}{ }^{\prime}\right)}{4 \pi\left(v_{M}-v_{M}{ }^{\prime}\right)}$ \\
Interferometry & $D \cong \frac{\Delta \phi \lambda^{2}}{4 \pi \Delta \lambda}$ \\
\hline
\end{tabular}

Table 3. Measurement uncertainty comparison

\begin{tabular}{cc}
\hline Methods & Distance \\
\hline Triangulation & $1 \mu \mathrm{m}$ \\
Pulse Telemetry & $5 \mathrm{~m} @ f_{c l k}=30 \mathrm{MHz}$ \\
Phase Comparison Telemetry & $0,557 \mathrm{~mm} @$ \\
Interferometry & $v_{M}=30 \mathrm{MHz}$ \\
& $0.15 \mu \mathrm{m}$ \\
\hline
\end{tabular}

\section{Conclusions}

The laser measurement technologies have many applications both at civil and military level - topographic surveying, 2D and 3D scanning, robots, UAVs, guided missiles, among others. The study of the three methods discussed in this paper thus plays an important role in the future development of more complex methods, for example, with the addition of 2 or 3 of the basic methods in a more complex single method.

Each method is preferably used to measure distances in a range of values. To complement this paper, it is important to briefly address each method, commenting on the results of the simulation and its practical results, where applicable.

The triangulation method allows to determine distances or positions of objects through the triangle geometry. A study was conducted on how to measure distances by applying this method. In the specific case of this work, it was defined as the triangle base the distance between the detector and the laser. To achieve the measurement, we determined the angles of the triangle sides, which interconnected the detector with the dice, and the laser with the dice, so as to have the maximum power received at the detector. In order to get a correct measurement, these two angles have to be equal. With one side and two angles of a triangle we can determine all the other sides and, thereby, determine the desired distance.

We conducted a simulation in the programming software Wolfram Mathematica 10.3 Student Edition that, in addition to measuring the distance from the detector to the target, joined several measures in order to show the 
surface of the 5 dots face of a dice. The result of this simulation confirms the theory, that is, applying the theoretically studied geometry, we obtained the surface of face 5 of the dice at the correct distance.

To prove the theoretical and simulation results, we made two experiments with the triangulation method. The first was a test to check whether the method application worked at a distance of $50 \mathrm{~cm}$ and $70 \mathrm{~cm}$, having a mirror as a target. It was a success. Thus, we made a second experiment with a dice at about $70 \mathrm{~cm}$ from the detector. This time, the goal was to scan across the whole 5 dots face of the dice to observe its surface, inserting all distances in a program developed with software Wolfram Mathematica 10.3 Student Edition. The results confirm the theoretical and simulation results for the obtained surface corresponds to the surface of the 5 dots face of the dice in the correct distance.

The method of pulse telemetry is based on determining the time-of-flight it takes a light pulse to travel from a transmitter to the target and then back to the detector. We have conducted a study on how to measure distances by applying this method. Using the same programming software, we created a program that simulates the application of this method to the distances measurement of approximately $50 \mathrm{~m}$. There was obtained a result which confirms the studied theory. The obtained measurement errors are expected and are duly substantiated, proving that this method has exceptional capabilities to measure long distances (up tens of meters) but has major limitations at reduced distances. With the increase in the frequencies of the clock that controls the counting, it is possible to reduce the minimum distance at which the method is effective. In the future, with the development of technology and the consequent improvement of the integrated circuits that can support higher frequencies, it will be possible to improve the performance of this method in distances that are smaller than a meter. Although we have not made any experiment with this method, we studied an experimental setup and developed a PCB with the help of EAGLE 7.2.0 software, that will allow to apply this method to distances measurement in a future work.

The method of phase comparison telemetry detects the light consistently reflected by the target to get the optical phase shift between the emitted and received signal. We proceeded with a study that explains how to measure distances by applying this method. Using the programming software mentioned above, we created a program that simulates the application of this method in measuring distances of about $50 \mathrm{~cm}$. We got the expected result compared to the theoretical results, as duly explained in the paper. Although we have not made any experiment with this method, we studied an experimental setup and developed a PCB with the help of EAGLE 7.2.0 software, that will allow to apply this method to distances measurement in a future work.

In the method of interferometry, the distance is measured by the phase difference between two waves, where one of the waves is known. We proceeded with a study about how to measure distances by applying this method. Using the programming software mentioned above, we created a program that simulates the application of this method to measure distances of about $5 \mu \mathrm{m}$ (with $1 \mathrm{~cm}$ reference). The result was consistent with the theory studied. It is a complex method for it involves distance measurements in the order of nanometers, and it is also costly due to the interferometer, which usually consists of first surface mirrors, a laser and a detector. Therefore, we haven't made any physical experiment to test the method. However, the method is properly described and simulated in the paper. Consequently, it is proposed as a future work the development of an interferometer based on the theoretical and simulation results of this paper.

In short, the paper objectives were achieved. As a future work, besides the development of the physical experiments mentioned above, it is proposed to proceed with a study on the possibility of combining the studied methods, in order to obtain one or more applications that include a measurement system capable of operating from nanometers to kilometers. In the current military reality, it is increasingly intended to take the conventional soldier out of the battlefield, which will be achieved by replacing Man by machines, such as robots. An effective and robust measurement system built into a robot can be crucial in different tasks required on a mission, for example, determining the distance to an explosive device with precision, in order to keep people away from the site in a safety distance, among others.

\section{Acknowledgments}

This work was support by FCT, under IT, Project No. UID/EEA/50008/2013.

\section{References}

A Cabral, A., \& Rebordão, J. M. (2015). Metrologia Absoluta De Comprimento Por Interferometria De Varrimento De Frequência. Retrieved from http://www.spmet.pt/Eventos/Encontro2/Alexandre_Cabral.pdf

Amann, M. C., Bosch, T., Lescure, M., Myllyla, R., \& Rioux, M. (2001). Laser ranging: a critical review of usual techniques for distance measurement. Optical engineering, 40(1), 10-19. https://doi.org/10.1117/1.1330700 
Berkovic, G., \& Shafir, E. (2012). Optical methods for distance and displacement measurements. Advances in Optics and Photonics, 4(4), 441-471. https://doi.org/10.1364/AOP.4.000441

Hadni, A. (1993). Applications des lasers, principes optiques avec problèmes commentés: R. Farcy Masson, Paris (1993). 320 pp., 188 Fr.

Kikuta, H., Iwata, K., \& Nagata, R. (1986). Distance measurement by the wavelength shift of laser diode light. Applied optics, 25(17), 2976-2980. https://doi.org/10.1364/AO.25.002976

Martin, D. (2007). Obituaries. Theodore Maiman, 79, Dies; Demonstrated First Laser. Retrieved from http://www.nytimes.com/2007/05/11/obituaries/11maiman.html?_r=0

Nejad, S. M., \& Olyaee, S. (2006). Comparison of TOF, FMCW and phase-shift laser range-finding methods by simulation and measurement. Quart. J. Technol. Educ, 1, 11-18.

\section{Copyrights}

Copyright for this article is retained by the author(s), with first publication rights granted to the journal.

This is an open-access article distributed under the terms and conditions of the Creative Commons Attribution license (http://creativecommons.org/licenses/by/4.0/). 